\title{
Quantitative characterization of mechanically indented in vivo human skin in adults and infants using optical coherence tomography
}

Pin-Chieh Huang

Paritosh Pande

Ryan L. Shelton

Frank Joa

Dave Moore

Elisa Gillman

Kimberly Kidd

Ryan M. Nolan

Mauricio Odio

Andrew Carr

Stephen A. Boppart 


\title{
Quantitative characterization of mechanically indented in vivo human skin in adults and infants using optical coherence tomography
}

\author{
Pin-Chieh Huang, ${ }^{a, b}$ Paritosh Pande, ${ }^{b}$ Ryan L. Shelton, ${ }^{b}$ Frank Joa, ${ }^{c}$ Dave Moore, ${ }^{c}$ Elisa Gillman, ${ }^{c}$ \\ Kimberly Kidd, ${ }^{c}$ Ryan M. Nolan, ${ }^{b}$ Mauricio Odio, ${ }^{c}$ Andrew Carr, ${ }^{c}$ and Stephen A. Boppart ${ }^{a, b, d, e, k}$ \\ aUniversity of Illinois at Urbana-Champaign, Department of Bioengineering, Urbana, Illinois, United States \\ bUniversity of Illinois at Urbana-Champaign, Beckman Institute for Advanced Science and Technology, Urbana, Illinois, United States \\ 'The Procter and Gamble Company, Cincinnati, Ohio, United States \\ dUniversity of Illinois at Urbana-Champaign, Department of Electrical and Computer Engineering, Urbana, Illinois, United States \\ eUniversity of Illinois at Urbana-Champaign, Department of Internal Medicine, Urbana, Illinois, United States
}

\begin{abstract}
Influenced by both the intrinsic viscoelasticity of the tissue constituents and the time-evolved redistribution of fluid within the tissue, the biomechanical response of skin can reflect not only localized pathology but also systemic physiology of an individual. While clinical diagnosis of skin pathologies typically relies on visual inspection and manual palpation, a more objective and quantitative approach for tissue characterization is highly desirable. Optical coherence tomography (OCT) is an interferometry-based imaging modality that enables in vivo assessment of cross-sectional tissue morphology with micron-scale resolution, which surpasses those of most standard clinical imaging tools, such as ultrasound imaging and magnetic resonance imaging. This pilot study investigates the feasibility of characterizing the biomechanical response of in vivo human skin using OCT. OCT-based quantitative metrics were developed and demonstrated on the human subject data, where a significant difference between deformed and nondeformed skin was revealed. Additionally, the quantified postindentation recovery results revealed differences between aged (adult) and young (infant) skin. These suggest that OCT has the potential to quantitatively assess the mechanically perturbed skin as well as distinguish different physiological conditions of the skin, such as changes with age or disease. @ 2017 Society of PhotoOptical Instrumentation Engineers (SPIE) [DOI: 10.1117/1.JBO.22.3.034001]
\end{abstract}

Keywords: optical coherence tomography; image analysis; skin; biomechanics; dermatology; edema.

Paper 160781SSR received Nov. 14, 2016; accepted for publication Feb. 9, 2017; published online Mar. 1, 2017.

\section{Introduction}

The biomechanical response of skin can often provide useful information about human health, such as the interindividual variability in hydration level, structural biomechanical properties, or pathological state. ${ }^{1-5}$ While the viscoelastic characteristics of skin may be altered after photodamage or in the presence of cancer, ${ }^{1-3}$ the overall mechanical response following a largescale localized indentation may reveal other systemic physiological conditions. ${ }^{4,5}$ Additionally, aging also plays an important role in the biomechanical response of the skin due to the wellknown age-induced structural and functional variations that occur in collagen and elastin.

Despite the importance of the evaluation of the tissue biomechanical response, there is yet no standard medical device or set of metrics developed for noninvasive, quantitative assessment of the mechanical response of human skin. Several commercially available devices have enabled the evaluation of the extensibility, elasticity (ability of recovery after deformation), stiffness (resistance to the change of shape), and viscous-component of the skin. ${ }^{6,7}$ However, the fact that they rely on suctioninduced deformation of the skin surface has often limited their region of assessment to the outer mechanical compartment of

*Address all correspondence to: Stephen A. Boppart, E-mail: boppart@illinois .edu the skin. Additionally, the spatiotemporal deformation inside the tissue cannot be directly monitored.

Optical coherence tomography (OCT) is a biomedical imaging technique that allows noninvasive detection of crosssectional morphology of biological tissues at micron-scale resolution, which surpasses many clinical imaging techniques, such as ultrasound imaging and magnetic resonance imaging. ${ }^{8}$ The 1- to 2-mm penetration depth along with the cellular-level resolution of OCT makes it an attractive technique for numerous dermatology investigations. ${ }^{9}$ Motivated by this, we present in this paper quantitative metrics based on OCT images, which are used to monitor the in vivo biomechanical response of skin (both the mechanically perturbed change and the time-evolved alterations of the tissue) and infer age-dependent physiological variations in the human subjects. The OCT metrics proposed were based on skin surface geometry, tissue optical properties, and tissue morphology (using texture analysis) and were derived from two main regions of the skin, namely the skin surface and the dermal skin (which we denoted as "in-tissue" throughout this paper).

The in-tissue OCT features were primarily obtained from the dermal layer, which contains blood vessels and collagen and dominates the mechanical behavior of skin. While the collagen meshwork and elastin fibers provide the main mechanical

1083-3668/2017/\$25.00 @ 2017 SPIE 
support and elastic recoil function, ${ }^{10}$ the ground substance located in the extrafibrillar space is responsible for the viscous property of deformation. ${ }^{11}$ The dermo-epidermal junction (DEJ), where the epidermal and dermal cells are "glued" via the basement membrane, is the interface between the epidermis and dermis. ${ }^{12}$ Therefore, the DEJ serves as an important boundary to be identified during data processing to ensure that the in-tissue OCT features were extracted from the dermis. To evaluate the performance of the proposed OCT metrics, two groups of human subjects with inherently different age-dependent biophysical and physiological conditions, namely adults and infants, were recruited in this pilot study. Both the indentation-induced alteration of the tissue and the postindentation recovery were tracked over time using the quantitative OCT metrics. The effectiveness of the OCT metrics in distinguishing adult from infant skin based on the indentation-induced biomechanical response was subsequently investigated in this paper.

The main contributions of this study are discussed below. First, to the best of our knowledge, texture analysis has only been performed on OCT data for tissue classification, especially for diseased tissues, but it has not yet been reported for evaluation or temporal tracking of the mechanically perturbed state of tissue. In addition, although mechanically induced changes of optical properties are widely recognized, a quantitative investigation of the postindentation residual effect on in vivo human skin with different ages has yet to be studied using OCT. Finally, to our knowledge, this is the first time that OCT has been utilized to examine the biomechanical responses from both infant and adult human skin, with quantitative comparative analysis performed on the pre- and postperturbed, and recovering skin over time.

\section{Background}

\subsection{Biomechanical Response of Skin, Physiology, and Age}

Alterations in systemic physiological conditions could be reflected in the biomechanical response of skin. For instance, the imbalance of fluid pressure, decreased drainage capacity, and increased permeability of the vasculature may lead to fluid accumulation within the extracellular space. ${ }^{13}$ By utilizing palpation or localized compression along with visual inspection, the physician can clinically evaluate the mechanical response of skin tissue, which can facilitate the detection of fluid retention or edema (an accumulation of interstitial fluid at the millimeter scale that is common in the lower extremities) and infer both local-regional and systemic diseases, such as chronic venous insufficiency, deep venous thrombosis, lymphedema, heart failure, and diabetes. ${ }^{14,15}$ Additionally, it is widely recognized that aging can also influence the morphology and functions of the skin (such as those that occur in collagen and elastin) and, hence, could also affect the overall biomechanical response of the skin. Structurally, aged skin exhibits reduced dermal thickness, collagen content, ground substances (mainly composed of water and glycosaminoglycans), and vascularity (particularly observed in the papillary dermis). ${ }^{16-18}$ Functionally, decreased rates of dermal clearance of fluids, vascular responsiveness, and moisture content of the stratum corneum were also reported. ${ }^{16-18}$ Adult skin and infant skin have demonstrated different properties. Compared to adult skin, the collagen in the upper reticular dermis of infants is thinner and less dense than in adults. Moreover, the level of skin hydration of older infants ( 3 to 12 and 8 to 24 months) was observed to be significantly higher than that of adults. ${ }^{19}$ The above-mentioned agedependent changes in skin could contribute to an alteration in both the intrinsic stiffness and the time-dependent fluid redistribution behavior. ${ }^{11,16}$ To date, the diminished resilience and elastic recovery after deformation of aged skin have been widely recognized. ${ }^{11,16,18}$ In particular, it has been observed that the recovery time of mechanically depressed skin of younger adults (minutes) is greatly different from that of older adults $(>24 \mathrm{~h}) .^{20}$

Applying a millimeter-scale deformation on human skin tissue affects not only the skin surface geometry but also the optical properties of skin tissue due to the temporary change in architectural morphology and interstitial fluid resulting from the applied indentation. Mechanically induced changes in optical properties have been revealed previously using OCT. Studies have shown an elevation of the backscattered intensity amplitude within the skin specimen due to the compression-induced local fluid transport. ${ }^{21}$ In fact, when a pressure gradient is introduced to the tissue, the unbound water or interstitial fluids are expelled aside, which decreases the absorption coefficient of the skin, especially when the light source is close to $1450 \mathrm{~nm}$ (where water has high absorption peak). ${ }^{21}$ In addition, mechanical compression can lead to an increase in optical tissue homogeneity, closer packing of tissue components, and a decrease of tissue thickness at the indented site. ${ }^{22}$ As a result, the index mismatch in refractive index is reduced, and the optical attenuation diminishes; hence, a greater backscattered intensity and penetration depth are observed. ${ }^{22,23}$ Mechanical compression has also been applied with optical clearing, and the prolonged clearing effect on in vivo human tissue even after the release of a localized stress has been reported. ${ }^{21,22}$ In addition, compressioninduced alterations of OCT intensity contrast within tissues has been shown to be effective for distinguishing between ex vivo inflamed and tumor-bearing rectal tissues. ${ }^{24}$

\subsection{Mechanical Compression and Tissue Morphology}

Upon indentation, morphological alterations within the skin tissue, which can affect the size, quantity, and distribution of the scatterers, could also occur and, hence, alter the OCT speckle patterns. A number of researchers have investigated methods to differentiate or classify pathological tissues based on the speckle pattern or statistics. Based on OCT signal intensity, Fourier or autocorrelation analysis detects the periodic pattern of the tissue structures. ${ }^{25}$ Basic statistics, such as the statistical moments and Gamma fitting of the intensity histogram, have also been applied for tissue characterization. ${ }^{26-28}$ Commonly performed on OCT images, texture analysis often takes advantage of other statistical features that are both intensity sensitive and directionally sensitive, such as the co-occurrence matrices ${ }^{29}$ and the gray-scale run-length matrices. ${ }^{30}$ In this paper, fractal analysis was utilized to characterize the intensity distribution within the tissue. The central concept of fractal analysis is to reveal the statistical self-similarity and geometrical complexity of an object based on the box-counting dimension. Previously, fractal analysis has been carried out in OCT images and has demonstrated its capability of distinguishing various types of tissue components within ex vivo arterial tissues and cancerous tissues. $^{31,32}$ 


\section{Materials and Methods}

\subsection{Human Subjects}

Two groups of subjects with distinct age differences were recruited to participate in this study: eight Caucasian female adults (20 to 33 years of age, two pregnant and six nonpregnant) and eight Caucasian infants ( 9 to 18 months of age, four females and four males). All the adult participants and the legal guardians of the infant participants provided written, informed consent before participating in the study. The study protocol was reviewed and approved by a Procter and Gamble (P\&G) clinical research review panel and was determined to be of minimal risk. The study was conducted with oversight from $\mathrm{P} \& \mathrm{G}$ 's clinical personnel in a manner consistent with Good Clinical Practice principles and procedures. The pregnant adults were excluded from the study due to well-known physiological changes in skin, such as the presence of edema, exhibited during pregnancy. ${ }^{33}$ For the adult group, the indentation sites were selected on the volar forearm (a quarter of the wrist-crease length measured from the elbow crease), while, for the infants, the indentation was induced on the lateral thigh (approximately halfway between the knee and the buttock). The two body sites were selected due to their comparable scale of circumferences, which would result in a similar scale of displacements applied to the skin, as the strain exertion was fixed in this study. For the adults, the body mass index (BMI) and body fat percentage (BF $\%$ ) were also obtained; the former was calculated based on the height and weight of the subject and the latter was measured by a body fat analyzer (Model HBF-306, Omron Healthcare, Inc., Illinois). Demographics of the subjects are included in Tables 1 and 2.

\subsection{Indentation Procedures and Devices}

For all subjects, the targeted indentation locations were marked prior to the application of the localized loading to ensure the image locations were kept the same at all times. Before indentation, baseline OCT data were acquired. Subsequently, indentation was applied on in vivo human subjects by a customized polymer-based indenter [Fig. 1(a)]. Plastic indenters with a dimension shown in Fig. 1(b) were machined, and an elastic band (Seraket ${ }^{\circledR}$ Automatic Tourniquet 48300-770, Propper manufacturing Co., Inc., New York) was used to hold the

Table 1 Demographics of human adult subjects.

\begin{tabular}{lcccccc} 
Subject & $\# 1$ & $\# 2$ & $\# 3$ & $\# 4$ & $\# 5$ & $\# 6$ \\
\hline Image site & $\begin{array}{c}\text { Volar forearm } \\
\text { Race }\end{array}$ & & & & & \\
Sex & Caucasian & & & & & \\
Age (year) & 20 & 28 & 30 & 30 & 32 & 33 \\
BMl & 19.6 & 29.3 & 15.6 & 31.7 & 20.8 & 29.7 \\
BF \% & 19.3 & 35.8 & 9.2 & 38.4 & 23.9 & 35.5 \\
Circf. (cm) & 22.3 & 26.8 & 20.3 & 26.4 & 24.5 & 27.2 \\
\hline
\end{tabular}

Note: BMI indicates the body mass index, BF \% indicates the body fat percentage, Circf. indicates circumference, and $\mathrm{F}$ indicates female.
Table 2 Demographics of human infant subjects.

\begin{tabular}{lcccccccc} 
Subject & $\# 1$ & $\# 2$ & $\# 3$ & $\# 4$ & $\# 5$ & $\# 6$ & $\# 7$ & $\# 8$ \\
\hline Image site & Lateral thigh & & & & & & & \\
Race & Caucasian & & & & & & & \\
Sex & $\mathrm{M}$ & $\mathrm{M}$ & $\mathrm{M}$ & $\mathrm{M}$ & $\mathrm{F}$ & $\mathrm{F}$ & $\mathrm{F}$ & $\mathrm{F}$ \\
Age (month) & 16 & 17 & 18 & 18 & 9 & 13 & 13 & 15 \\
Circf. (cm) & 26.7 & 26.4 & 24.6 & 28.6 & 28.3 & 26.2 & 24.5 & 24.2 \\
\hline
\end{tabular}

Note: Circf. indicates circumference, $\mathrm{M}$ indicates male, and $\mathrm{F}$ indicates female.

indenter in place for $3 \mathrm{~min}$ [Fig. 1(c)]. The strain applied was fixed at $\sim 16.7 \%$, so the length of the elastic band was adjusted according to the circumferences of the body sites of the subjects. Due to the large strain applied $(>10 \%)$, a red marking and indentation could be visually observed on the skin upon the release of the indenter [Fig. 1(d)]. After indentation, the OCT probe was immediately placed at the indented skin site for image acquisition at the following time-points: immediately ( $<10 \mathrm{~s}$, for simplicity, this is denoted as "0-min") and 1, 2, 3, 4, and 5 min after the indentation (postindentation). The observation period of the skin recovery was chosen at the minute-scale based on the finding of a previous study. ${ }^{20}$ During the imaging session, the subjects' forearms were rested on a metal "hand stand" covered with a soft fabric to keep the skin surface flat and stable at all times [Fig. 1(e)].

\subsection{Optical Coherence Tomography Instrumentation and Data Collection}

A commercial spectral domain OCT system (center wavelength $\sim 1300 \mathrm{~nm}$, Telesto SD-OCT, Thorlabs, Inc., Germany) with an axial resolution of $\sim 6.5 \mu \mathrm{m}$ in air and a lateral resolution of $\sim 15 \mu \mathrm{m}$ was utilized. A 36-mm focal length objective (LSM03, Thorlabs, Inc., Germany), which provided a Rayleigh range of $\sim 106 \mu \mathrm{m}$, was used for imaging. The B-scans acquired from the OCT system had dimensions of 512 pixels $(\sim 2.5 \mathrm{~mm})$ along the depth and 1024 pixels $(\sim 6 \mathrm{~mm})$ along the lateral direction. During imaging, an axial line scan rate of $\sim 5.5 \mathrm{kHz}$ was utilized and a sensitivity of $\sim 111 \mathrm{~dB}$ was achieved. The signal-to-noise ratio was $77 \mathrm{~dB}$, and the signal fall-off measured between $10 \%$ and $90 \%$ of the imaging depth was $3.5 \mathrm{~dB}$. The $1 / e$ penetration depth of light into the nonindented skin tissues was characterized to be $\sim 0.40 \pm 0.07 \mathrm{~mm}$ (average \pm standard deviation) in optical distance.

A customized polymer-based attachment was mounted at the end of the rigid OCT scanner to enable handheld imaging and user-friendly assessment of the body sites of interest (i.e., volar forearm for adults and lateral thigh for infants), as well as to keep the working distance fixed between the tissue and the lens [Figs. 2(a) and 2(b)]. To minimize the possibility of introducing vascular occlusion or other sources of perturbation near the target sites of interest, the physical dimensions of the probe attachment [Fig. 2(a)] were designed so that the diameter of the aperture $(\sim 2.7 \mathrm{~cm})$ was significantly larger than the width of the indenter tip $(\sim 0.75 \mathrm{~mm})$. In addition, the ring-shaped edge around the aperture was designed to have a larger area 

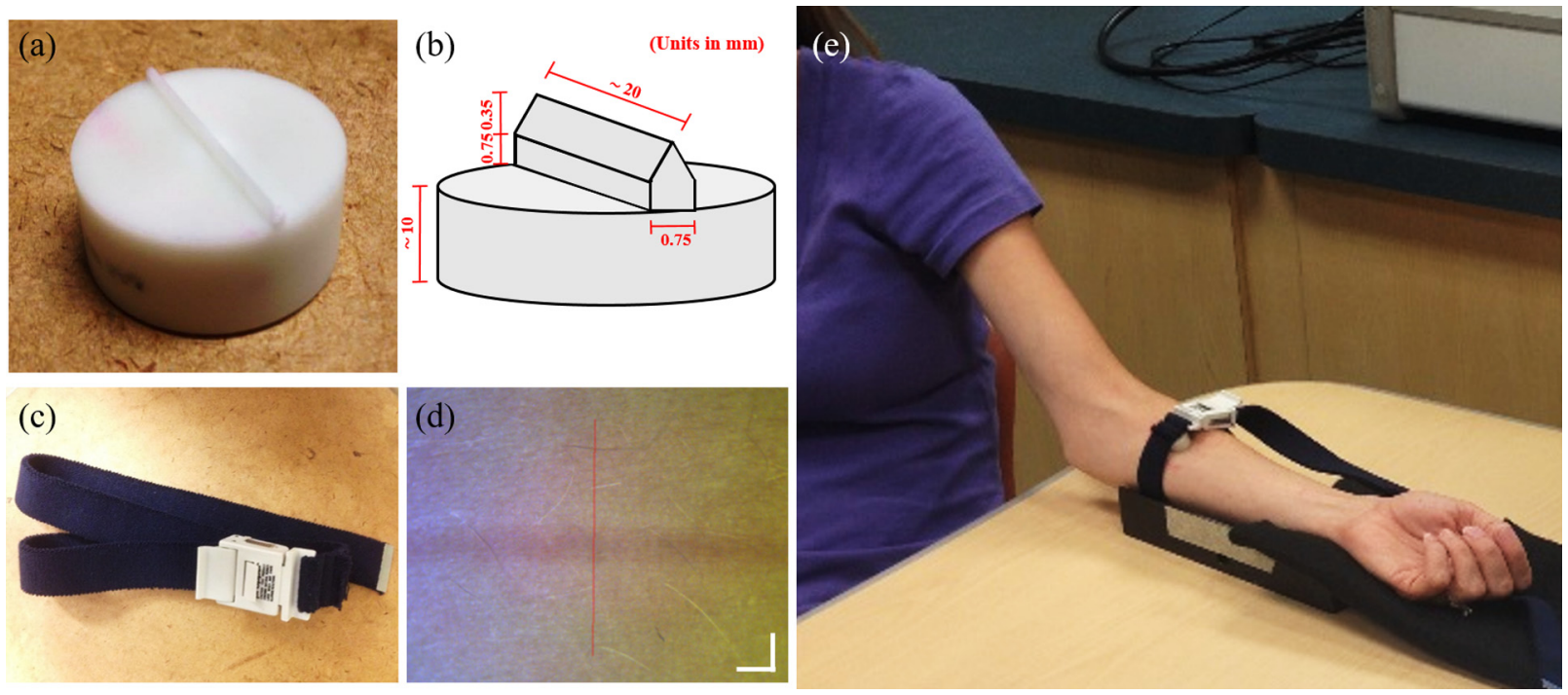

Fig. 1 Indentation device and setup. (a) The photograph of the custom-made polymer indenter, along with (b) its geometry and dimensions. The measured dimensions are illustrated in millimeters (mm). (c) The elastic strap used to hold the indenter in place. (d) A representative surface image of the in vivo human skin tissue, immediately $(<10 \mathrm{~s})$ after the release of the indenter. The scale bars in both the vertical and horizontal directions both represent $1 \mathrm{~mm}$. The vertical red line indicates the scanning location for OCT images. (e) An adult subject undergoing the 3-min indentation procedure.
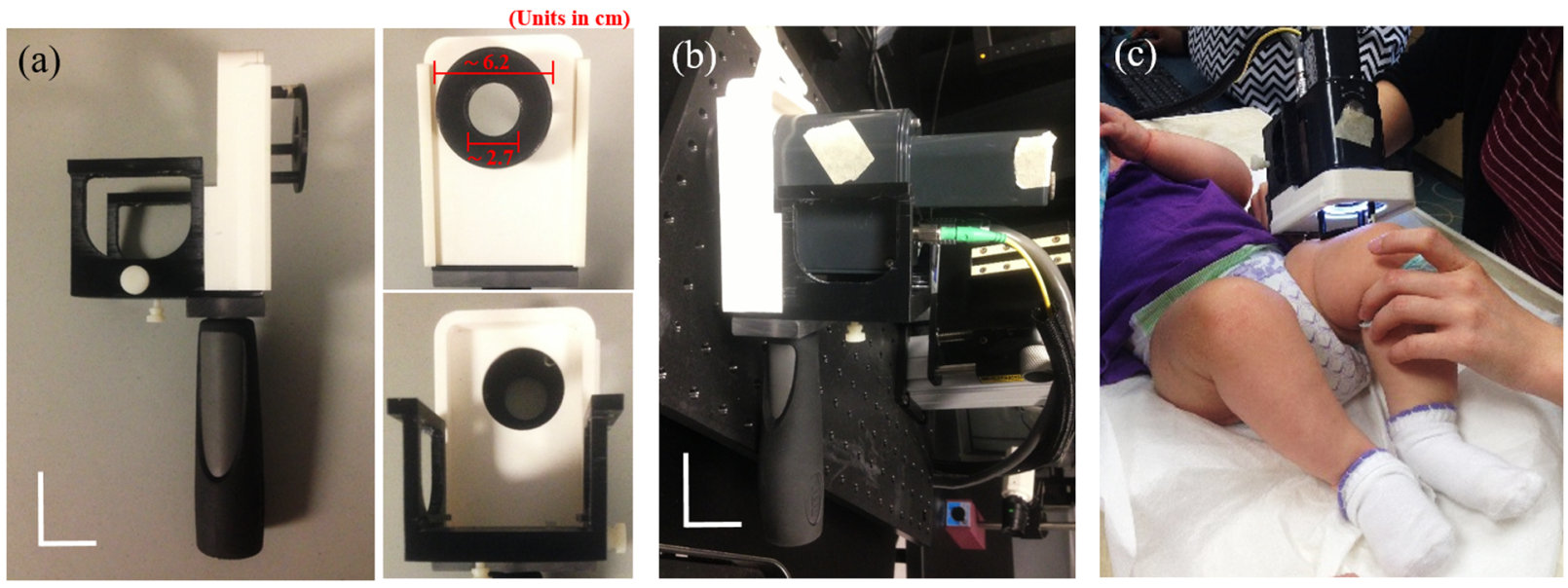

Fig. 2 The handheld probe and the imaging process. (a) The design and the dimensions of the handheld attachment. The photographs were taken from (left) the side, (top right) the side facing the skin, and (bottom right) the side carrying the rigid scanner head. The dimensions of the aperture are illustrated in centimeters (cm). (b) By mounting the rigid scanner to the attachment, (c) handheld OCT imaging was enabled. The vertical and horizontal scale bars represent 4 and $3 \mathrm{~cm}$, respectively.

$\left(\sim 24.5 \mathrm{~cm}^{2}\right)$ so that the amount of pressure applied at the edge would be reduced.

Standard OCT data processing on the raw data was performed using the commercial software available from Thorlabs (OCT 3.0.7, Thorlabs, Inc., Germany). The OCT data were saved as 8-bit gray scale images. These intensity-based images were then used as the input for extracting the different OCT features used in this study, where all the quantitative processing was performed on MATLAB $^{\circledR}$ (R2016a, MathWorks, Massachusetts). Multiple frames were acquired on each patient until at least three stable frames of the images were obtained. Eventually, three frames were utilized as image inputs per time-point (i.e., pre- and postindention at 0, 1, 2, 3, 4, and $5 \mathrm{~min}$ ) per patient for quantitative analysis. As a result, the analyses included three replicate measures $(n=3)$ for each patient and $N=8$ (number of subjects) for each group, leading to a total number of frame $N_{\text {total }}=24$ per scenario. Representative OCT images of the adult and the infant skin are shown in Fig. 3.

\subsection{Image Preprocessing}

To correct for imaging artifacts due to the presence of surface skin hair and misalignments induced by inevitable subject and operator motion, the OCT images were preprocessed. To assist artifact removal and image alignment, a skin surface boundary was first identified by determining the axial location at which the cumulative intensity along the depth was greater than the 

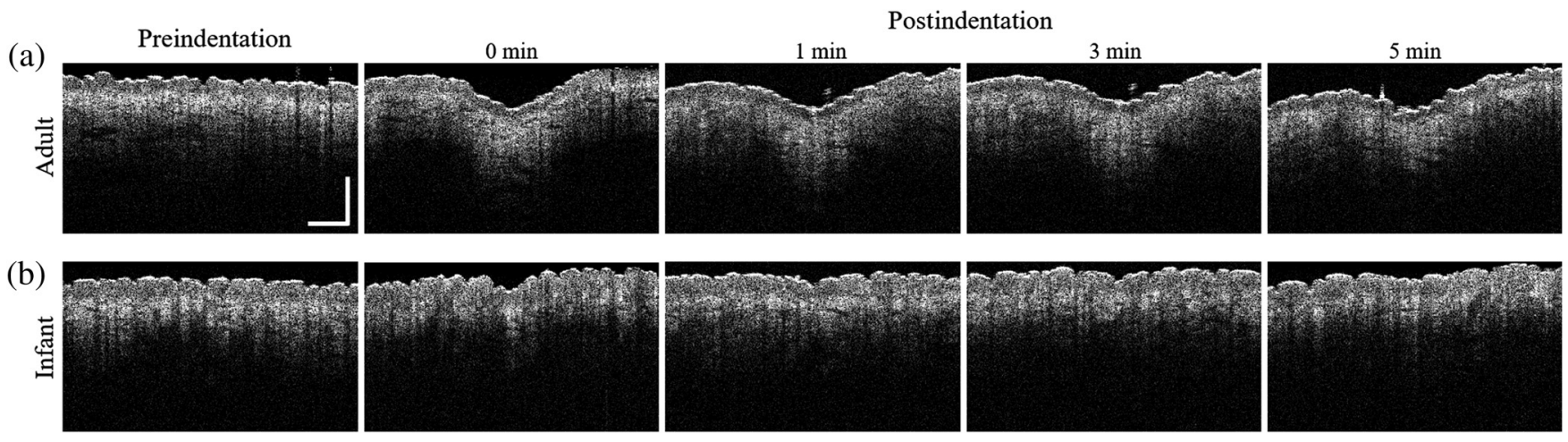

Fig. 3 Representative OCT images of in vivo human skin before and after indentation. (a) Representative adult forearm skin and (b) representative infant thigh skin. Both the horizontal and vertical bars indicate $500 \mu \mathrm{m}$.

empirically determined threshold value. Subsequently, artifacts were removed from the images [Figs. 4(a)-4(g)]. The surface hair artifacts (above the surface) were identified by finding the lateral location where the averaged intensity within the "air ribbon" region was above a threshold [Figs. 4(c) and 4(d)], and the shadow artifacts (beneath the surface) were identified by searching for the lateral position where the intensity slope beneath the skin surface was below a threshold [Figs. 4(e) and 4(f)]. Finally, image alignment in both lateral and axial directions was implemented. Laterally, the "indented site," as opposed to the nonindented skin regions surrounding the indented site, was determined by the local minimum (valley) of the surface boundary [Fig. 4(i)]. To ensure that the in-tissue OCT features could be extracted from the dermis, the data were axially aligned to the DEJ, which was identified based on the maximum intensity found in each moving-average filtered A-scan [Fig. 4(k)]. Detailed preprocessing procedures are described in the Appendix.

\subsection{Image Analysis and Quantitative Metrics}

To characterize the deformed tissue, quantitative OCT metrics were developed based on three aspects: the change in skin surface geometry, optical property, and dermis morphology. The latter two metrics are "in-tissue" parameters.

\subsubsection{Surface-geometry-based metrics}

Similar to other commercially available devices that measure skin biomechanical properties, ${ }^{6,7}$ the residual deformation percentage (RD \%) was defined based on the geometric alteration of the skin surface. The RD \% was calculated as the deformation that remained at the center indented site $x_{\text {indented }}\left(D_{\text {residual }}\right)$, normalized by the initial applied displacement $\left(D_{\text {applied }}\right)$, expressed as $\frac{D_{\text {residual }}}{D_{\text {applied }}} \times 100 \%$.

As illustrated in Fig. 4(j), the residual or remaining deformation $\left(D_{\text {residual }}\right)$ was obtained around $x_{\text {indented }}$ by measuring the distance from the skin surface to the average height of the two raised peaks immediately adjacent to the indented site [the red filled dots shown in Fig. 4(j)]. The raised peaks were determined by local maximum detection at the two neighboring regions of $x_{\text {indented }}$, each of which had a lateral range of 256 pixels $(\sim 1.5 \mathrm{~mm}$ in width). The same measurement of distance was performed at 5 A-scan locations $(\sim 20 \mu \mathrm{m})$ around $x_{\text {indented }}$, whose average represents $D_{\text {residual }}$.

\subsubsection{Optical-property-based metrics}

For the in-tissue features, the aligned images [based on the DEJ, Fig. 4(k)] were utilized so that all quantitative features were extracted from the dermis. An intensity ratio ( $I$ ratio) was obtained by normalizing the averaged intensity at the indented site $\left(I_{\text {indented }}\right)$ with those from the nonindented sites $\left(I_{\text {nonindented }}\right)$, given as $\frac{I_{\text {indented }}}{I_{\text {nonindented }}}$.

The term $I_{\text {indented }}$ denotes the averaged intensity within the region-of-interest at the indented site $\left(\mathrm{ROI}_{\text {indented }}\right)$; and $I_{\text {nonindented }}$ was an average of the intensity within the two nonindented regions $\left(\mathrm{ROI}_{\text {nonindented }}\right)$, each of which was selected to be 145 pixels $(\sim 850 \mu \mathrm{m})$ away from $\mathrm{ROI}_{\text {indented }}$ in the lateral direction [Fig. 4(1)]. Each ROI had a lateral width of 68 pixels $(\sim 400 \mu \mathrm{m})$ and an axial range of $250 \mu \mathrm{m} \times \frac{D_{\text {applied }}}{1 \mathrm{~cm}}$. Note that the applied strain was fixed; hence, $D_{\text {applied }}$ varied with the circumference of either the forearm or the thigh of each subject. The axial range of the ROI was limited to $250 \mu \mathrm{m}$, approximately where single scattering dominates in a highly scattering tissue. $^{34}$

\subsubsection{Dermis-morphology metrics}

Dermal morphology was quantified based on the fractal dimensions (FDs) computed at the regions defined in Sec. 3.5.2. The fractal dimension ratio ( $F D$ ratio) was defined as $\frac{\mathrm{FD}_{\text {indented }}}{\mathrm{FD}_{\text {nonindented }}}$, where $\mathrm{FD}_{\text {indented }}$ and $\mathrm{FD}_{\text {nonindented }}$ denote an average of the one-dimensional FD (1-D FD) obtained within $\mathrm{ROI}_{\text {indented }}$ and $\mathrm{ROI}_{\text {nonindented, }}$, respectively [Fig. 4(1)]. For example, if the width of the $\mathrm{ROI}_{\text {indented }}$ spanned across 68 columns, the 1-D FD would first be calculated from each column, and an average of the 68 1-D FD values would be used to represent FD $_{\text {indented. }}$. In each ROI, the 1-D FD of each column was obtained based on a 1-D box-counting method. The total length-of-interest of each column $\left(l_{0}\right)$ was determined by the highest power-of-two value less than or equal to the axial range of the original ROI. For instance, if the original ROI had an axial range of 35 pixels, $l_{0}$ would be $2^{5}=32$ pixels.

Within each ROI, each column was segmented into several intervals, each with a length of $l_{i}$, where $l_{i}=\frac{l_{0}}{2^{i}}$ was the length of the interval at the $i$ 'th iteration. The number of iteration was determined by $l_{0}$ so that a minimum length of 1 pixel was achieved for $l_{i}$ eventually. For instance, a $l_{0}$ of $2^{5}$ pixels would result in $i=1,2, \ldots, 5$ and $l_{5}=1$. The number of 
Artifact removal

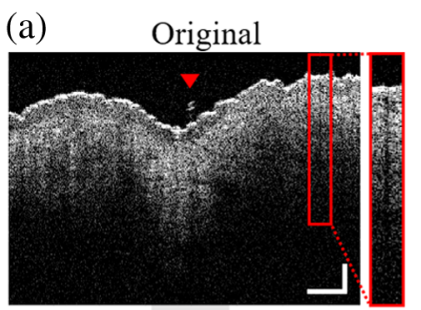

(g) Artifact removed
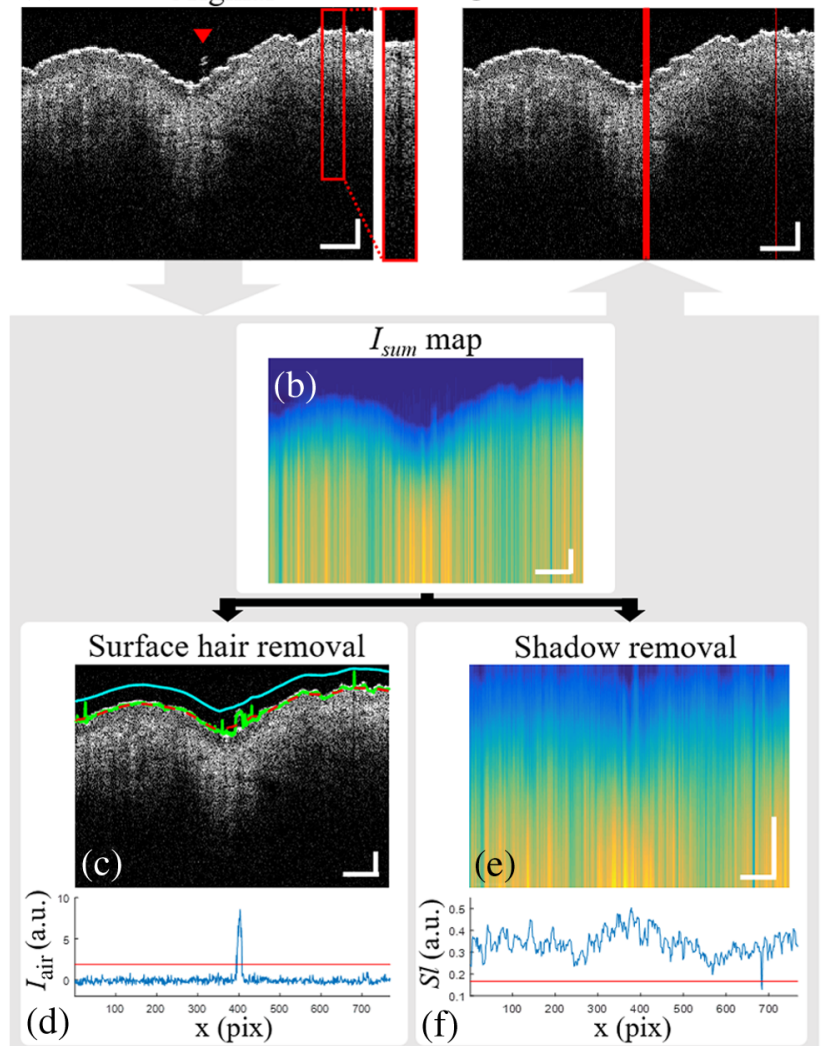

\section{Image alignment}

(h) Unaligned

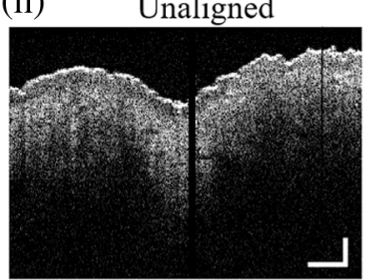

(i)

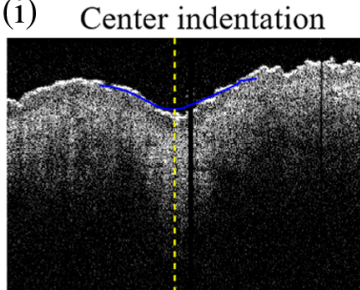

(k) DEJ estimation

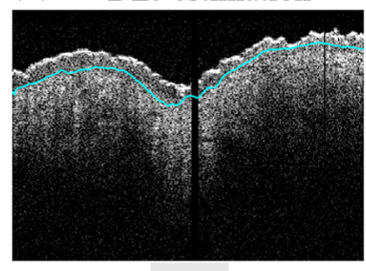

(j)

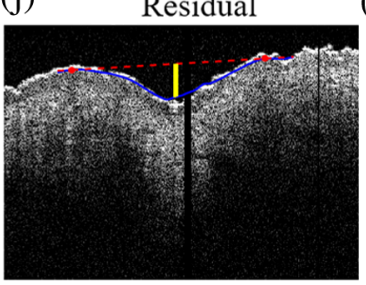

(1) ROIs defined on aligned image

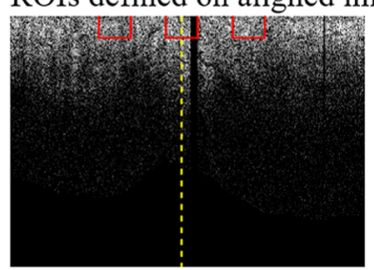

Fig. 4 Data preprocessing for (a-g) artifact removal and (h-l) image alignment. (a) Original image with (red triangle) surface hair and (red squared box) shadow artifacts, with a zoomed-in image of the latter shown on the right. (b) Moving average filtered $I_{\text {sum }}$ map. (c) The initial estimation of the surface including the surface hair, $S_{i}$, (green trace), and excluding the surface hair, $S_{f}$, (red dashed trace). The upper boundary of the "air ribbon" region is also identified (cyan trace). (d) The corresponding $\bar{l}_{\text {air }}(X)$ at each column (blue plot) and the threshold (red line). (e) Aligned and smoothed (b) $I_{\text {sum }}$ map with a depth of 150 pixels. (f) The corresponding $S I(X)$ at each column (blue plot) and the threshold (red line). (g) A combined result after removing the surface reflection (b-d) and shadow artifacts (b, e, f). Image alignment was performed on the artifact-removed unaligned image (h). (i) The center indentation site (yellow dashed line), determined by valley detection at the neighboring region (blue lines). (j) The $D_{\text {residual }}$ (solid yellow line) determined near the center indented site as the distance between the skin surface (blue lines) and the interpolated line (red dashed) between the two neighboring peaks (filled red dots). (k) Determined DEJ (cyan trace), which was used for (I) axial alignment. The defined center $\mathrm{ROI}_{\text {indented }}$ and side $\mathrm{ROI}_{\text {nonindented }}$ (red boxes). The center indented site ( $x_{\text {indented }}$ ) (yellow dashed line) determined in (i). The vertical and horizontal scale bars correspond to 200 and $500 \mu \mathrm{m}$, respectively. Detailed preprocessing procedures are described in Appendix.

intervals that included any pixel with an intensity greater than the threshold value $\left(I_{\text {threshold }}\right)$ was denoted as $N_{i}$. Note that $I_{\text {threshold }}$ was adaptively determined so that only the predominated signal, instead of the noise floor, was picked up. Initially, the FD as a function of various threshold intensity values was obtained. Eventually, the threshold value that yielded an FD of $75 \%$ of the maximum achievable FD [typically 1, as shown in Fig. 5(b)] was determined as $I_{\text {threshold }}$. The 1-D FD of each column was determined as the slope of $\log \left(N_{i}\right)$ over $\log \left(\frac{1}{l_{i}}\right)$, fitted via linear least square regression [Fig. 5(a)]. The averaged $R$-squared values for the fitting at all ROIs for all data are $\sim 0.93$. The FD infers the self-similarity in the sense that each part of the curve reflects the entire curve except for a scaling factor difference. ${ }^{32}$ A more disordered or complex pattern is expected to give rise to a higher FD value. $^{31}$

\subsection{Statistical Analysis}

Given the relatively small sample size and nonnormal distribution of the data, nonparametric tests were used. A two-sided sign test was performed on the nonindented and the 0 -min postindented skin from the same group of subjects, and a two-sided Wilcoxon rank sum test was performed on the infant and the adult skin at the postindentation scenarios. For both tests, the significance level was set to $5 \%$.

\section{Results and Discussions}

\subsection{Indentation-Induced Alterations}

As shown in Fig. 6, before the application of any localized compression, the surface geometrical feature, quantified by RD \%, exhibited a value close to zero (a flat skin surface) for both 

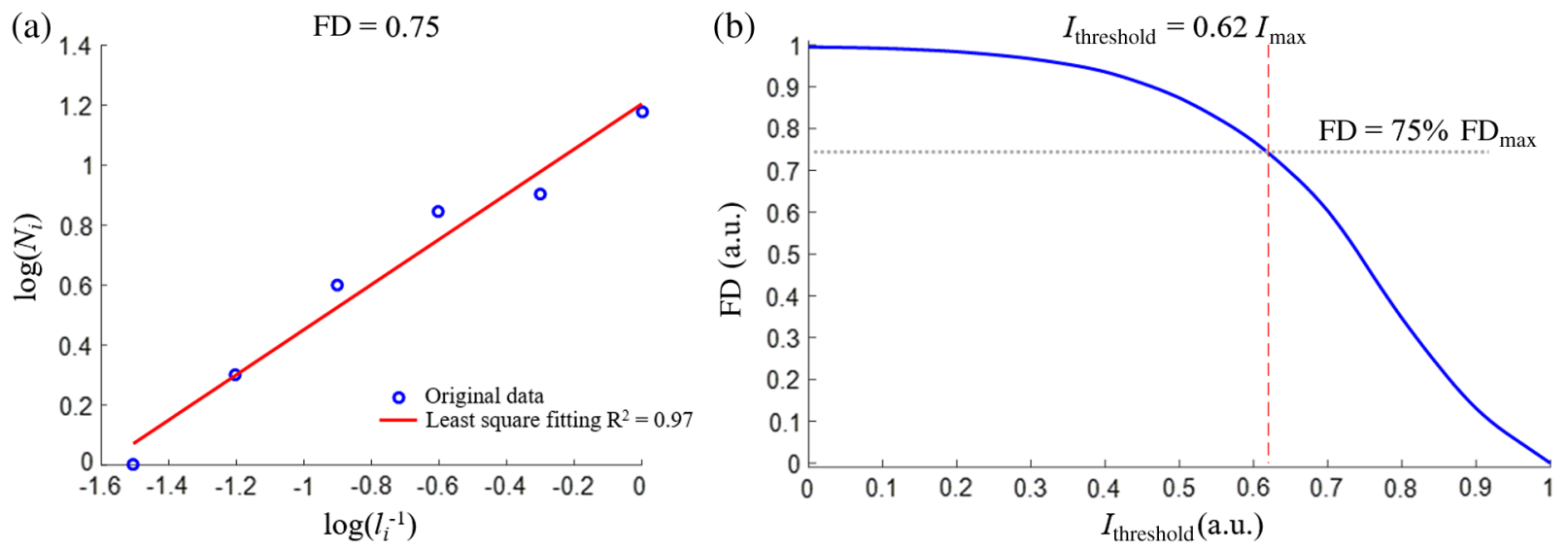

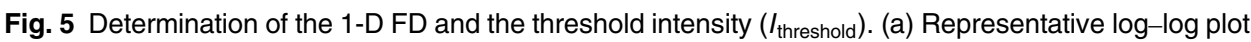
of number of boxes $\left(N_{i}\right)$ versus the box size $\left(I_{i}\right)$ for calculation of FD for one column. The slope obtained by linear least square fitting is the FD. (b) The $I_{\text {threshold }}$ was defined as the intensity value that resulted in an FD equivalent of $75 \%$ of the maximum FD.
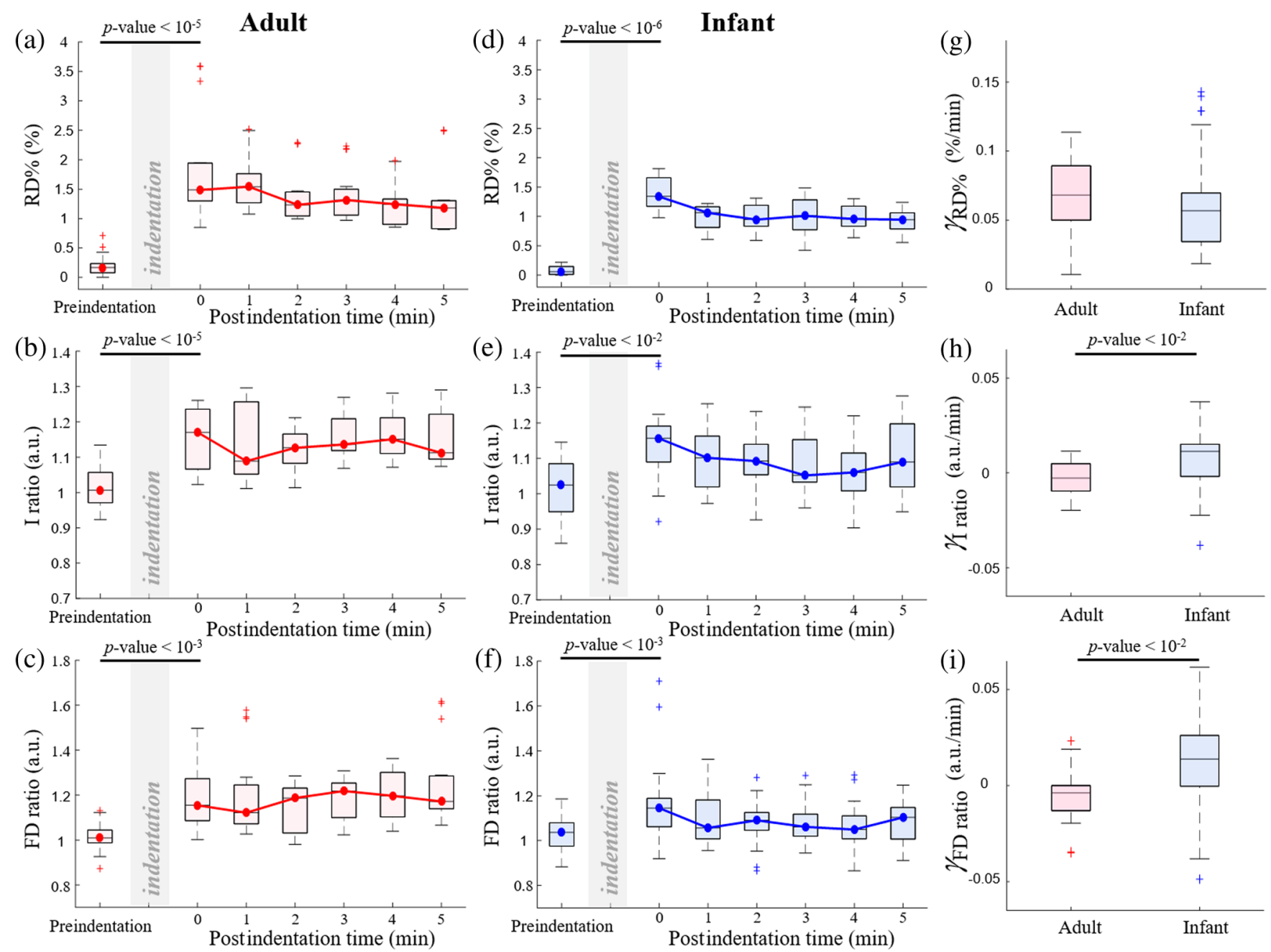

Fig. 6 Results from the adults and the infants, quantified based on the developed OCT features (a-f) and the postindentation recovery coefficients $\gamma(\mathrm{g}-\mathrm{i})$. Box plots of the adult skin response at the preindentation and 0-5 min postindentation time-points for the (a) RD \%, (b) / ratio, and (c) FD ratio parameters. Box plots of the infant skin responses at the preindentation and 0-5 min postindentation time-points for the (d) RD\%, (e) I ratio, and (f) FD ratio parameters. Shown in the third column are the box plots of (g) $\gamma_{\mathrm{RD} \%}$, (h) $\gamma_{/ \text {ratio }}$, and (i) $\gamma_{\mathrm{FD} \text { ratio }}$ for both the adults (red) and the infants (blue). $\mathrm{N}_{\text {total, adult }}=18$. $\mathrm{N}_{\text {total, infant }}=24$. 
datasets. Immediately after the removal of the indenter (0-min postindentation), visible deformation was seen in the OCT images, while positive RD \% values were detected for both the adult and the infant datasets $\left(p\right.$-value $<10^{-5}$ and $10^{-6}$, respectively). This indicated a delayed recovery of the skin, which was similar to the observation reported in previous studies. ${ }^{5,20}$ Likewise, the $I$ ratio and the FD ratio were close to unity before the indentation, suggesting little variation in the optical and morphological features across lateral locations of the dermal tissue. However, upon the release of the indentation (0-min postindentation), a significant increase was observed for both the $I$ ratio ( $p$-values $<10^{-5}$ for adults and $10^{-2}$ for infants) and FD ratio ( $p$-value $<10^{-3}$ for both adults and infants) metrics. This indicated that the average intensity, along with the averaged 1-D FD, have a greater value at the indented site as compared to those of the nonindented nearby regions. The observed increase of the backscattered intensity was in agreement with previous studies, showing the compressioninduced intensity change within soft tissues. ${ }^{21,22}$ In addition, the increased FD value revealed after indentation suggested an increased complexity of the distribution of intensity in OCT images, possibly due to the denser distribution of the tissue scatterers caused by the indentation.

\subsection{Postindentation Recovery}

After the release of the indenter, the three OCT parameters were tracked over time to monitor the rebound of the skin for both the adult forearm and the infant thigh tissues. Illustrated in Figs. 6(a)-6(f), both the adult and the infant datasets exhibited a decrease in the metric values at the postindentation timepoints, indicating that the deformed skin was gradually recovering toward its initial preindented state. This could be a combined result of displacement recovery from the elastic tissue components (e.g., collagen, elastin, etc.) and the redistribution of the unbound fluids of the ground substances over time, reflecting the viscous property of skin. To quantify the recovery trend over time $(t)$, a weighted least squares fitting based on an exponential function $\left(e^{-\gamma t}\right)$ was performed on the three OCT metrics to obtain the recovery coefficient $\gamma$. A higher, positive value of $\gamma$ represents a faster rate of recovery toward the preindented state. The $\gamma$ values were calculated for each individual dataset collected from each human subject, and the pool of $\gamma$ values was subsequently utilized for statistical analysis.

Box plots of the recovery coefficients for both the adult and the infant datasets are shown in Figs. 6(g)-6(i), where $\gamma_{\mathrm{RD} \%}$, $\gamma_{I \text { ratio }}$, and $\gamma_{\mathrm{FD} \text { ratio }}$ represent the recovery coefficients of the RD \%, I ratio, and FD ratio, respectively. Comparing the results from the adult skin to that of the infant skin, significant differences were observed for both in-tissue parameters, $\gamma_{I \text { ratio }}$ and $\gamma_{\mathrm{FD} \text { ratio }}(p$-values $<0.01)$, but not for $\gamma_{\mathrm{RD} \%}(p$-value $=0.6)$. Both dermal recovery coefficients from the infant skin were positive (the medians of $\gamma_{I \text { ratio }}$ and $\gamma_{\text {FD ratio }}$ were $1.1 \times 10^{-2} / \mathrm{min}$ and $1.4 \times 10^{-2} / \mathrm{min}$, respectively), indicating a tendency of the dermal parameters to return toward the nondeformed states. However, the adult datasets exhibited recovery coefficients that were an order-of-magnitude smaller (the medians of $\gamma_{I \text { ratio }}$ and $\gamma_{\text {FD ratio }}$ were both at a scale of $10^{-3} / \mathrm{min}$ ), implying negligible temporal variation within $5 \mathrm{~min}$ after the release of the localized loading. This suggests that the rebound of the fluids and the deformable tissue components within the 5-min postindentation period was less appreciable than in the infant group, which agrees with the findings of other studies that aged skin has a decreased rate of dermal clearance of fluids and vascular responsiveness. ${ }^{16,17}$ The fact that significant differences were observed in the adult and the infant groups based on the distribution of $\gamma_{I \text { ratio }}$ and $\gamma_{\mathrm{FD} \text { ratio }}$ suggests that the in-tissue parameters (based on the optical intensity and morphology of the dermis) could be more effective than the surface geometrical metrics in distinguishing the age-induced variations of the biomechanical response within the first 5 -min postindentation period. This demonstrates the potential merits of using OCT imaging for quantitative characterization of the biomechanical response of skin tissue.

While these differentiating results were acquired within the 5-min postindention measurement period, it is interesting to note that none of the OCT metrics (both surface geometry and intissue-based) completely returned to their initial values within the experimental measurement time period. Additionally, all OCT features at the 5-min postindentation were different from the preindentation features ( $p$-value $\leq 0.001$ for the adults and 0.06 for the infants), suggesting a high likelihood that mechanical equilibrium had not yet been fully reached after 5-min postindentation. Future studies will extend this temporal characterization to longer time points, which will further improve fitting of the recovery kinetics and provide additional data on potential differences between adult and infant skin conditions.

\section{Conclusions and Future Directions}

In this study, three metrics (the RD\%, I ratio, and FD ratio) were developed based on OCT image data to quantitatively characterize the mechanical response of human skin upon application and release of an external force. The proposed metrics were applied to in vivo human skin data from both the adult forearm and the infant thigh, where significant differences were found between the pre- and postindentation ( 0 -min) time periods in both groups of subjects. While most of the commercially available devices for skin measurement rely on the displacement confined to the skin surface, ${ }^{6,7}$ this study has demonstrated the feasibility of implementing the optical backscattered intensity and the morphology at the dermal skin as in-tissue metrics to assist in the quantification of the tissue biomechanical response. Additionally, the postindentation recovery coefficients, quantified by exponentially fitting the in-tissue OCT features (i.e., $\gamma_{I \text { ratio }}$ and $\left.\gamma_{\mathrm{FD} \text { ratio }}\right)$ within the first $5 \mathrm{~min}$ after indentation, demonstrated the possibility of further differentiating adult from infant skin. These findings suggest that the proposed OCT features, particularly those extracted from the dermis, could be utilized to assess the age-dependent physiological variations based on the 5-min postindentation recovery trend of the skin tissue. However, the authors do realize that there was a relatively short period of recovery time after the release of skin indentation, primarily because of the need to minimize the imaging time for the infant subjects. Future studies will extend the time coverage of the measurements so that a full recovery can be observed for both the adults and the infants and further characterization on the biomechanical properties of the skin can be provided. In addition, the relatively small sample size and the limited number of temporal sampling points in the current datasets are noted. To further reduce the margin of error and increase the power and confidence levels, an expanded number of human subjects in each group will be necessary for future studies. Once a lengthened measurement time and a denser temporal sampling are 
achieved, a thorough investigation of various curve fitting models and approaches for the postindentation recovery can be conducted in a systematic manner.

Following this initial study, several improvements can be recommended for future investigations. First, fully automatic surface detection and image segmentation approaches (e.g., Ref. 35) can be implemented for more objective preprocessing procedures. Additionally, transparent indenters and force sensors can be implemented so that the changes during indentation can be visualized and investigated, enabling the assessment of additional biomechanical parameters, such as the Young's modulus and viscosity of in vivo tissue. While the physiological differences between the adult and infant skin have been reflected in the spatiotemporal skin response after an indentation at a fixed strain, it would also be interesting to explore the correlation of the mechanical response between the two groups under a range of indentation strains or forces. Moreover, finite element models can be developed with consideration for the nonlinear viscoelastic or poroelastic behaviors of the sample, ${ }^{36,37}$ and simulation results can be compared with empirical results, such as for the three-dimensional strain distribution of the tissue over time. Other OCT metrics, such as using the lateral spatial distribution of informative features to infer the lateral translocation of the fluid components, can conceivably be developed too.

This paper demonstrated the feasibility of using OCT-based quantitative metrics to characterize the biomechanical response of mechanically deformed skin beyond the linear elastic region, which could be indicative of age-dependent biomechanical and physiological conditions. While the deformation at the skin surface could be detected by OCT with micron-scale precision, it is the indentation-induced alterations of the OCT intensity and the FD in the dermal tissue that could be utilized to distinguish the time-dependent mechanical behavior of aged (adult) and young (infant) skin. This highlights the merits of using OCT as it provides simultaneous assessment of both the optical intensity and the tissue structure within in vivo skin, from which the proposed in-tissue parameters were derived. In the future, the same quantitative analysis can be carried out on OCT images of skin with different dermatological pathologies to explore the feasibility of using these metrics for clinical diagnosis. Additionally, these OCT-based metrics were currently applied to characterize the overall biomechanical response of the in vivo skin, which has a complex nature resulting from multiple biophysical factors. Hence, other OCT-related and optical microscopy techniques may also be incorporated to better understand the specific underlying biophysics. For instance, optical coherence elastography can be used to evaluate the viscoelastic properties of tissue components, and Doppler OCT or optical microangiography can be applied to monitor the vascular distribution. ${ }^{1-3,38,39}$ Optical coherence microscopy (OCM) can be used to detect structural, biomechanical, and hemodynamics of skin at the cellular level. ${ }^{40,41}$ Moreover, OCM can be integrated with multiphoton and fluorescence lifetime imaging microscopy to provide molecular contrast, infer cellular metabolism state, and monitor the cell dynamics in skin tissue. ${ }^{42,43}$ Visualization of molecular information of skin can also be assessed via nonlinear interferometric vibrational imaging. ${ }^{44}$ While the correlation between the above-mentioned information and the overall biomechanical response (quantified with the proposed OCT metrics) may be further explored for improving our basic scientific understanding of the skin, a collection of these measurements could potentially provide a more informative, objective, and quantitative tool for clinical diagnosis of localized and systemic diseases.

\section{Appendix: Image Preprocessing Details}

\section{A.1 Surface Identification}

Surface identification was performed as follows. First, a spatial map of the summed intensity was generated by representing each pixel at each location as $I_{\text {sum }}\left(x, z_{n}\right)=\sum_{i=1}^{n} I\left(x, z_{i}\right)$, followed by a $5 \times 1$ moving average filtering [Fig. 4(b)]. Next, the initial surface boundary $\left(S_{i}\right)$ was determined by allocating the axial location at which the summed intensity was greater than an empirically determined threshold value. Finally, the surface boundary $\left(S_{f}\right)$ was determined by laterally moving average filtered $S_{i}$ with a $1 \times 40 \mathrm{kernel}$ [Fig. 4(c)].

\section{A.2 Artifacts Removal}

Surface skin hair (above the surface) and shadow (beneath the surface) artifacts were then removed as follows. First, above the skin surface, a two-dimensional (2-D) "air ribbon" region was defined with a thickness that was the distance between the surface hair and the skin surface (i.e., maximum distance between $S_{i}$ and $S_{f}$ ). The intensity of the air ribbon was averaged along the axial direction and detrended along the transverse direction, resulting an $\bar{I}_{\text {air }}(X)$ as a function of A-scan locations $X=\left\{x_{j}\right\}$, where $x_{j}$ denotes the $j$ 'th A-scan [Fig. 4(d)]. The location of the surface skin hair was then determined as the A-scans with $\bar{I}_{\text {air }}\left(x_{j}\right)$ greater the mean of $\bar{I}_{\text {air }}(X)$ plus 2.5 times the standard deviation of $\bar{I}_{\text {air }}(X)$, provided that skin hair was present within the frame. On the other hand, the processing of shadow artifacts was performed based on the intensity slope $[\mathrm{Sl}(X)]$ quantified at each column of the 2-D $I_{\text {sum }}$ map, where $I_{\text {sum }}$ was aligned to the skin surface $\left(S_{f}\right)$ and $S l(X)$ was quantified by fitting a line across a depth of 150 pixels from the surface [Figs. 4(e) and 4(f)]. Finally, the locations of shadow artifacts were identified as the A-scans that produced a slope smaller than the minimum of $S l(X)$ plus $10 \%$ of the range (i.e., the maximum minus minimum) of $S l(X)$. Using the abovementioned approaches, the surface hair and shadow artifacts were effectively identified and removed. Note that manual inspection was performed on the processed results.

\section{A.3 Image Alignment}

Image alignment was performed next. Lateral image alignment was implemented to identify the "indented site" as opposed to the nonindented surrounding locations of the skin. The center indented site $\left(x_{\text {indented }}\right)$ was determined as the lateral location at which the local minimum (valley) was observed on the detrended, moving average filtered skin surface curvature $\left(S_{f}\right)$, where the valley detection was performed within a lateral range of 342 pixels $(\sim 2 \mathrm{~mm})$ centered at a manually estimated center location [Fig. 4(i)]. Axial image alignment was performed so that the data could align to the DEJ, and, hence, the in-tissue OCT features could be extracted from the dermis. To determine the DEJ, the tissue region (starting from 20 pixels beneath the $S_{f}$ ) was first moving-average filtered with a $5 \times 1$ kernel at each A-scan, followed by allocating the maximum intensity within each A-scan. Finally, a $1 \times 30$ moving average filtering process was performed laterally (across all A-scans) 
and resulted in the final outcome, defined as the DEJ [Fig. 4(k)].

\section{Disclosures}

Dr. S. A. Boppart discloses a financial interest in Diagnostic Photonics, Inc. Dr. S. A. Boppart, Dr. R. L. Shelton, and R. M. Nolan disclose a financial interest in PhotoniCare, Inc. Neither of these companies, however, supported this study.

\section{Acknowledgments}

This project was supported in part by the $\mathrm{P} \& \mathrm{G}$ Company and the National Institutes of Health (No. R01 EB013723). Dr. S. A. Boppart receives royalties from the Massachusetts Institute of Technology for patents related to OCT. Additional information on OCT and its applications can be found at: http://biophotonics. illinois.edu.

\section{References}

1. B. F. Kennedy, K. M. Kennedy, and D. D. Sampson, "A review of optical coherence elastography: fundamentals, techniques and prospects," IEEE J. Sel. Top. Quantum Electron. 20(2), 272-288 (2014).

2. X. Liang, V. Crecea, and S. A. Boppart, "Dynamic optical coherence elastography: a review," J. Innov. Opt. Health Sci. 3(4), 221-233 (2010).

3. X. Liang and S. A. Boppart, "Biomechanical properties of in vivo human skin from dynamic optical coherence elastography," IEEE Trans. Biomed. Eng. 57(4), 953-959 (2010).

4. G. P. Berry et al., "The spatio-temporal strain response of oedematous and nonoedematous tissue to sustained compression in vivo," Ultrasound Med. Biol. 34(4), 617-629 (2008).

5. K. G. Brodovicz et al., "Reliability and feasibility of methods to quantitatively assess peripheral edema," Clin. Med. Res. 7(1-2), 21-31 (2009).

6. P. Elsner, D. Wilhelm, and H. Maibach, "Mechanical properties of human forearm and vulvar skin," Br. J. Dermatol. 122(5), 607-614 (1990).

7. M. Gniadecka and J. Serup, "Suction chamber method for measuring skin mechanical properties: the dermaflex," in Handbook of NonInvasive Methods and the Skin, 2nd ed., G. B. E. Jemec, G. L. Grove, and J. Serup, Eds, Vol. 2, pp. 571-577, Taylor \& Francis, Boca Raton, Florida (2006).

8. D. Huang et al., "Optical coherence tomography," Science 254(5035), 1178-1181 (1991).

9. T. Gambichler, V. Jaedicke, and S. Terras, "Optical coherence tomography in dermatology: technical and clinical aspects," Arch. Dermatol. Res. 303(7), 457-473 (2011).

10. Z. Zaidi and S. W. Lanigan, "Skin: structure and function," in Dermatology in Clinical Practice, pp. 1-15, Springer, New York City (2010).

11. P. G. Agache et al., "Mechanical properties and Young's modulus of human skin in vivo," Arch. Dermatol. Res. 269(3), 221-232 (1980).

12. J. G. Marks and J. J. Miller, Lookingbill and Marks' Principles of Dermatology, Elsevier Health Sciences, Amsterdam, Netherlands (2013).

13. C. Porth, Essentials of Pathophysiology: Concepts of Altered Health States, Lippincott Williams \& Wilkins, Philadelphia (2011).

14. K. P. Trayes et al., "Edema: diagnosis and management," Am. Fam. Physician. 88(2), 102-110 (2013).

15. R. J. Johnson et al., "Potential role of sugar (fructose) in the epidemic of hypertension, obesity and the metabolic syndrome, diabetes, kidney disease, and cardiovascular disease," Am. J. Clin. Nutr. 86(4), 899-906 (2007).

16. N. A. Fenske and C. W. Lober, "Structural and functional changes of normal aging skin," J. Am. Acad. Dermatol. 15(4), 571-585 (1986).

17. J. M. Waller and H. I. Maibach, "Age and skin structure and function, a quantitative approach (I): blood flow, $\mathrm{pH}$, thickness, and ultrasound echogenicity," Skin Res. Technol. 11(4), 221-235 (2005).
18. J. M. Waller and H. I. Maibach, "Age and skin structure and function, a quantitative approach (II): protein, glycosaminoglycan, water, and lipid content and structure," Skin Res. Technol. 12(3), 145-154 (2006).

19. G. N. Stamatas et al., "Infant skin physiology and development during the first years of life: a review of recent findings based on in vivo studies," Int. J. Cosmet. Sci. 33(1), 17-24 (2011).

20. W. L. Kydd, C. H. Daly, and D. Nansen, "Variation in the response to mechanical stress of human soft tissues as related to age," J. Prosthet. Dent. 32(5), 493-500 (1974).

21. A. A. Gurjarpadhye et al., "Effect of localized mechanical indentation on skin water content evaluated using OCT," J. Biomed. Imaging 2011 , 17 (2011).

22. K. V. Larin et al., "Optical clearing for OCT image enhancement and indepth monitoring of molecular diffusion," IEEE J. Sel. Top. Quantum Electron. 18(3), 1244-1259 (2012).

23. C. Drew, T. E. Milner, and C. G. Rylander, "Mechanical tissue optical clearing devices: evaluation of enhanced light penetration in skin using optical coherence tomography," J. Biomed. Opt. 14(6), 064019 (2009).

24. P. D. Agrba et al., "Compression as a method for increasing the informativity of optical coherence tomography of biotissues," Opt. Spectrosc. 107(6), 853-858 (2009).

25. A. M. Zysk and S. A. Boppart, "Computational methods for analysis of human breast tumor tissue in optical coherence tomography images," J. Biomed. Opt. 11(5), 054015 (2006).

26. S. Wang et al., "Three-dimensional computational analysis of optical coherence tomography images for the detection of soft tissue sarcomas," J. Biomed. Opt. 19(2), 021102 (2014).

27. A. A. Lindenmaier et al., "Texture analysis of optical coherence tomography speckle for characterizing biological tissues in vivo," Opt. Lett. 38(8), 1280-1282 (2013).

28. P. B. Garcia-Allende et al., "Morphological analysis of optical coherence tomography images for automated classification of gastrointestinal tissues," Biomed. Opt. Express 2(10), 2821-2836 (2011).

29. K. W. Gossage et al., "Texture analysis of speckle in optical coherence tomography images of tissue phantoms," Phys. Med. Biol. 51(6), 15631575 (2006)

30. P. Pande et al., "Automated classification of optical coherence tomography images for the diagnosis of oral malignancy in the hamster cheek pouch," J. Biomed. Opt. 19(8), 086022 (2014).

31. A. C. Sullivan, J. P. Hunt, and A. L. Oldenburg, "Fractal analysis for classification of breast carcinoma in optical coherence tomography," J. Biomed. Opt. 16(6), 066010 (2011).

32. C. Flueraru et al., "Added soft tissue contrast using signal attenuation and the fractal dimension for optical coherence tomography images of porcine arterial tissue," Phys. Med. Biol. 55(8), 23172331 (2010).

33. R. C. Wong and C. N. Ellis, "Physiologic skin changes in pregnancy," J. Am. Acad. Dermatol. 10(6), 929-940 (1984).

34. L. Scolaro et al., "Parametric imaging of the local attenuation coefficient in human axillary lymph nodes assessed using optical coherence tomography," Biomed. Opt. Express 3(2), 366-379 (2012).

35. M. R. N. Avanaki and A. Hojjatoleslami, "Skin layer detection of optical coherence tomography images," Optik-Int. J. Light Electron Opt. 124(22), 5665-5668 (2013).

36. E. B. Paul et al., "Nonlinear and poroelastic biomechanical imaging: elastography beyond Young's modulus," in Handbook of Imaging in Biological Mechanics, pp. 199-216, CRC Press, Boca Raton, Florida (2014).

37. S. Kalyanam, R. D. Yapp, and M. F. Insana, "Poro-viscoelastic behavior of gelatin hydrogels under compression-implications for bioelasticity imaging," J. Biomech. Eng. 131(8), 081005 (2009).

38. Y. H. Zhao et al., "Phase-resolved optical coherence tomography and optical Doppler tomography for imaging blood flow in human skin with fast scanning speed and high velocity sensitivity," Opt. Lett. 25(2), 114-116 (2000).

39. W. J. Choi, H. Wang, and R. K. Wang, "Optical coherence tomography microangiography for monitoring the response of vascular perfusion to external pressure on human skin tissue," J. Biomed. Opt. 19(5), 056003 (2014).

40. X. Liang, B. W. Graf, and S. A. Boppart, "In vivo multiphoton microscopy for investigating biomechanical properties of human skin," Cell. Mol. Bioeng. 4(2), 231-238 (2011). 
41. B. W. Graf and S. A. Boppart, "Multimodal in vivo skin imaging with integrated optical coherence and multiphoton microscopy," IEEE J. Sel. Top. Quantum Electron. 18(4), 1280-1286 (2012).

42. Y. Zhao et al., "Integrated multimodal optical microscopy for structural and functional imaging of engineered and natural skin," J. Biophotonics 5(5-6), 437-448 (2012)

43. Y. Zhao et al., "Longitudinal label-free tracking of cell death dynamics in living engineered human skin tissue with a multimodal microscope," Biomed. Opt. Express 5(10), 3699-3716 (2014).
44. W. A. Benalcazar and S. A. Boppart, "Nonlinear interferometric vibrational imaging for fast label-free visualization of molecular domains in skin," Anal. Bioanal. Chem. 400(9), 2817-2825 (2011).

Biographies for the authors are not available. 